University of Nebraska - Lincoln

DigitalCommons@University of Nebraska - Lincoln

Papers in Virology

Papers in the Biological Sciences

September 2003

\title{
Bovine herpes virus gD protein produced in plants using a recombinant tobacco mosaic virus (TMV) vector possesses authentic antigenicity
}

D. M. Pérez Filgueira

University of Nebraska - Lincoln

\section{P.I. Zamorano}

Instituto de Virología, Centro de Investigación en Ciencias Veterinarias y Agronómicas, INTA, CC25, 1712 Castelar, Buenos Aires, Argentina

M. G. Dominguez

Instituto de Virología, Centro de Investigación en Ciencias Veterinarias y Agronómicas, INTA, CC25, 1712 Castelar, Buenos Aires, Argentina

O. Taboga

Instituto de Biotecnología, Centro de Investigación en Ciencias Veterinarias y Agronómicas, INTA, CC25,

1712 Castelar, Buenos Aires, Argentina

M. P. Del Medico Zajac

Instituto de Virología, Centro de Investigación en Ciencias Veterinarias y Agronómicas, INTA, CC25, 1712

Fastelar Buenos Aires, Argentina

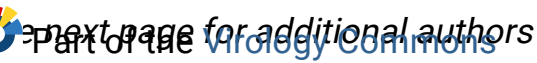

Pérez Filgueira, D. M.; Zamorano, P.I.; Dominguez, M. G.; Taboga, O.; Del Medico Zajac, M. P.; Puntel, M.; Romera, S. A.; Morris, Thomas Jack; Borca, M. V.; and Sadir, A. M., "Bovine herpes virus gD protein produced in plants using a recombinant tobacco mosaic virus (TMV) vector possesses authentic antigenicity" (2003). Papers in Virology. 2.

https://digitalcommons.unl.edu/bioscivirology/2

This Article is brought to you for free and open access by the Papers in the Biological Sciences at DigitalCommons@University of Nebraska - Lincoln. It has been accepted for inclusion in Papers in Virology by an authorized administrator of DigitalCommons@University of Nebraska - Lincoln. 


\section{Authors}

D. M. Pérez Filgueira, P.I. Zamorano, M. G. Dominguez, O. Taboga, M. P. Del Medico Zajac, M. Puntel, S. A. Romera, Thomas Jack Morris, M. V. Borca, and A. M. Sadir 


\title{
Bovine herpes virus gD protein produced in plants using a recombinant tobacco mosaic virus (TMV) vector possesses authentic antigenicity
}

\author{
D.M. Pérez Filgueira a,e,*,1, P.I. Zamorano ${ }^{\text {bee, }, \text {, M.G. Domínguez }}{ }^{\mathrm{b}}$, O. Taboga ${ }^{\mathrm{c}}$, \\ M.P. Del Médico Zajac ${ }^{\mathrm{b}}$, M. Puntel ${ }^{\mathrm{b}}$, S.A. Romera ${ }^{\mathrm{b}}$, \\ T.J. Morris ${ }^{\mathrm{a}}$, M.V. Borca ${ }^{\mathrm{d}, \mathrm{e}}$, A.M. Sadir ${ }^{\mathrm{b}, \mathrm{e}}$ \\ a School of Biological Sciences, University of Nebraska, Lincoln, NE 68588-0118, USA \\ b Instituto de Virología, Centro de Investigación en Ciencias Veterinarias y Agronómicas, \\ INTA, CC25, 1712 Castelar, Buenos Aires, Argentina \\ c Instituto de Biotecnología, Centro de Investigación en Ciencias Veterinarias y Agronómicas, \\ INTA, CC25, 1712 Castelar, Buenos Aires, Argentina \\ d Plum Island Animal Disease Center, USDA, Greenport, NY 11944, USA \\ e CONICET, Av. Rivadavia 1917, 1033 Buenos Aires, Argentina
}

Received 16 December 2002; received in revised form 8 April 2003; accepted 17 June 2003

\begin{abstract}
A tobacco mosaic virus (TMV)-based vector was utilized for expression of a cytosolic form of the bovine herpesvirus type 1 (BHV-1) protein glycoprotein D (gDc). Nicotiana benthamiana plants were harvested 7 days after inoculation with RNA transcripts derived from the TMV-gDc recombinant virus. Recombinant gDc protein of expected electrophoretic mobility accumulated in inoculated leaves to a concentration of about $20 \mu \mathrm{g} / \mathrm{g}$ of fresh leaf tissue. Oil-based vaccines were formulated with crude foliar extracts to immunize mice parentally. After a single injection, animals developed a sustained and specific response to both the isolated gD and native virus particles. Cattle vaccinated with the same gDc containing extracts developed specific humoral and cellular immune responses directed against both the viral gD and BHV-1 particles. Most importantly, animals vaccinated with the plant-produced gDc showed good levels of protection after challenge with the virulent BHV-1. Virus excretion was drastically reduced in these animals, reaching levels comparable to animals vaccinated with a commercial BHV-1 vaccine. The positive immunological characterization obtained for the gDc, indicated that an important part of the natural conformation was retained in the plant recombinant protein.
\end{abstract}

(C) 2003 Elsevier Ltd. All rights reserved.

Keywords: TMV-based vectors; Recombinant protein; Bovine herpes virus-1 (BHV-1)

\section{Introduction}

Plants represent one of the most abundant biomass sources for large-scale production of biological products. Several reviews on the application of this technology argue that it offers cost efficiency over more expensive fermentation or cell culture based methods [1-3]. Particularly in the vaccine field, several publications reported the development of transgenic plants expressing successful immunogens for oral or parenteral immunization [4-7]. Foreign proteins can also be produced in plants by transient expression using

\footnotetext{
* Corresponding author. Present address: Depto. Biotecnología, INIA-Madrid, Ctra. A Coruña Km.7, Madrid 28040, Spain.

Tel.: +34-91-347-6893; fax: +34-91-357-3107.

E-mail address: mperez@inia.es (D.M. Pérez Filgueira).

${ }^{1}$ Authors contributed equally to the results presented in this report.
}

plant virus-based vectors [8]. The ability of these vectors to achieve transient expression of a foreign gene product has some advantages over constitutive expression from a transgenic plant. Most notable is the fact that products of interest can be produced very rapidly after inoculation of mature plants with the additional benefit that the recombinant proteins can accumulate to high levels without appreciable degradation or toxic effects on the plant. Plant viral-based vectors have been successfully produced from a variety of plant RNA viruses and can be generally classified in those that can display foreign peptides fused to the coat proteins on the surface of viral particles [9] and those that are able to express whole recombinant proteins that accumulate within the plant, independently from other vector proteins [10]. Tobacco mosaic virus (TMV)-based expression vectors represent one of the more successful examples of this technology [11] and have been utilized for production of 
different therapeutic proteins, such as $\alpha$-trichosanthin [12] and tumor-specific single-chain antibodies [13]; and vaccine antigens, such as a malarial peptide [14] and the VP1 structural protein from foot-and-mouth disease virus (FMDV) [15]. We report here the use of the TMV-30B vector [16] which typifies a relatively stable family of TMV-based protein expression vectors-for the production in plants of a protein from bovine herpesvirus type 1 (BHV-1).

BHV-1 is the causative agent of a group of respiratory and reproductive disorders in cattle that is commonly referred to as infectious bovine rhinotracheitis [17]. The disease affects both adult and young animals and is of significant economic importance worldwide. Usual symptoms include rhinotracheitis, infectious pustular vulvovaginitis, enteritis, general respiratory disease and encephalitis, decreased milk production, weight loss and abortion in pregnant cows [18]. The BHV-1 vaccines in current use are formulated with either inactivated or modified live virus and both have a number of disadvantages. The inactivated vaccines are often poor immunogens and they have been shown to cause clinical disease if they are insufficiently inactivated [19]. Alternatively, live vaccines may induce immunosuppression [20] and complicate the process of discerning between vaccinated and infected animals. Alternative vaccination strategies involving the use of individual viral components such as glycoprotein $\mathrm{D}(\mathrm{gD})$ to induce protective immune responses to BHV-1 have been explored to overcome these problems [21-24].

In this report, the TMV-30B vector was utilized to produce a truncated form of the BHV-1 gD (cytosolic or gDc) protein in plants. Crude extracts of leaves inoculated with the recombinant virus were used to parentally immunize mice and cattle. We were able to show that these plant extracts induced both humoral and cellular-specific responses that recognized both the isolated protein and BHV-1 virus particles. Our results also demonstrated that the recombinant gDc could be stably produced in plants and that it retained antigenic integrity. More importantly, we showed that crude plant extracts containing the gDc protein had sufficient immunogenic potential to induce protection in the natural host against viral challenge.

\section{Materials and methods}

\subsection{Construction of the TMV-based expression vector carrying $B H V-1 g D c$}

The recombinant TMV-gDc vector was obtained from a variant of the TMV-30B expression vector [16] kindly provided by Dr. W.O. Dawson. Cloning of the foreign sequence into the TMV vector was as previously described [15]. The gDc sequence corresponding to nucleotides 54 through 1079 of the full-length $\mathrm{gD}$ was obtained by PCR from a pGem plasmid carrying the BHV-1 $g I V$ gene. The primers, gDc-PacI, (5'-ATTAATTAATGAGCTTGCCTACACCCGCGCCG-3') and gDc-PmeI ( $5^{\prime}$-GTTTAAACTCAGGCGTCGGGG GCCGCGGG-3'), were used to introduced start and stop codons at the extremes of the gDc sequence along with $P a c \mathrm{I}$ and PmeI restriction sites, respectively. The resulting fragment was then digested with $P a c \mathrm{I}$ and PmeI and inserted in the polylinker sequence of the TMV vector (Fig. 1). Integrity and correct insertion of the gDc sequence into the TMV-30b vector were confirmed by restriction analysis and nucleotide sequencing. The TMV-gDc plasmid was linearized by digestion with PstI and used as DNA template for the in vitro production of the infectious viral capped RNA following manufacturer instructions (Amplicap T7 transcription Kit, Epicentre Technology). Nicotiana benthamiana plants were mechanically inoculated on the upper surface of mature leaves using infectious RNA transcripts diluted in buffer containing $1 \%$ Na-pyrophosphate (pH: 9.0), $1 \%$ celite and $1 \%$ bentonite. Plants were kept in growth chambers $(16 \mathrm{~h}$ per day of light, $30^{\circ} \mathrm{C}$ day temperature, $24^{\circ} \mathrm{C}$ night temperature) until harvested. Inoculated foliar material was stored at $-20^{\circ} \mathrm{C}$ until utilized.

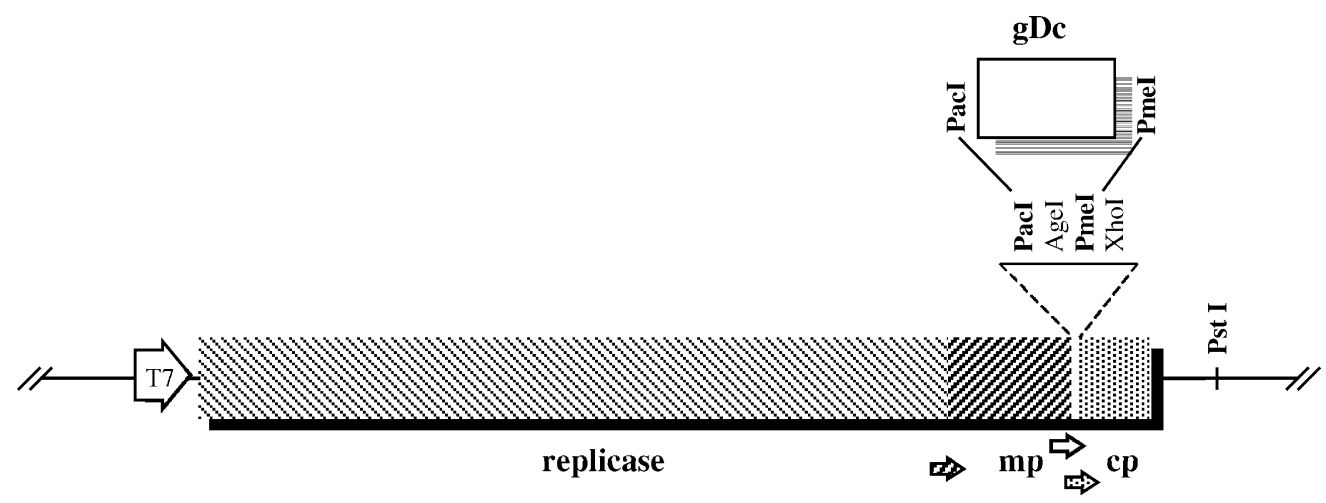

Fig. 1. Schematic structure of the TMV-gDc vector. The figure shows the location of the TMV-derived genes in the vector (the RNA-dependent RNA polymerase (replicase), the movement protein (mp) and the coat protein (cp)) and the gDc insert (1.05 kb) located between the PacI and PmeI sites in the polylinker sequence. Subgenomic promoters are indicated as arrows upstream of the corresponding genes as is the location of the T7 promoter and key restriction sites. 


\subsection{Western blot analysis of the TMV-gDc infected} leaves

Western blot analyses were performed on extracts from inoculated leaves using a polyclonal mouse antiserum specific for a recombinant $\mathrm{gD}$ produced in $E s$ chirichia coli $(g D-E c)$ [25]. Leaves were harvested at 7 days post-inoculation (dpi) and total protein was obtained from inoculated leaves by an organic extraction protocol following the manufacturer instructions (Tripure, Roche). The extracted proteins were separated in a $12.5 \%$ SDS polyacrylamide gel (PAGE) and transferred onto an ECL nitrocellulose membrane (Amersham). The membranes were blocked overnight with phosphate saline buffer (PBS)-Tween 20 (PBST), 4\% skim milk (blotto buffer) and incubated with a polyclonal mouse antiserum specific for the $g D$-Ec. Blots were then incubated with a HRP-labeled anti-mouse IgG antibody (Amersham) and developed with X-film (Kodak) using the ECL Western blotting detection system (Amersham). Concentration of the recombinant proteins was estimated from blots in a Biorad Fluor $\mathrm{S}$ MultiImager System using the Quantity One 4.0 software (BioRad).

\subsection{Animal immunizations}

\subsubsection{Mice}

Male BALB/c mice (6-8 weeks old) were used for vaccination. Animals received a single dose of an oil-based vaccine formulated with incomplete Freund adjuvant (IFA) by the intra-peritoneal (i.p.) route. Fresh plant tissue was macerated in the presence of liquid nitrogen, resuspended phosphate saline buffer (PBS) $\mathrm{pH} 7.5$ and emulsified in the oily phase. Mice received $0.2 \mathrm{ml}$ of this vaccine containing $1 \mathrm{~g}$ of fresh leaf tissue/ml. A control group of animals were also vaccinated with $0.2 \mathrm{ml}$ of a conventional oil BHV-1 vaccine (conv. vac.) formulated in IFA and containing inactivated BHV-1 with a previous titer of $10^{7.5}$ tissue-culture infective dose $50 \%\left(\mathrm{TCID}_{50}\right) / \mathrm{ml}$. Animals were bled at different times post-vaccination.

\subsubsection{Cattle}

Twelve to fourteen-month-old Holstein and Angus $x$ Hereford cows were utilized in the immunization experiments. Animals were selected based on their negative serology for BHV-1. Cattle were immunized at days 0, $21,45,70,120,150$ and 195 using $5 \mathrm{ml}$ of a similar vaccine as described for mice. Half of the dose was injected by the intra-muscular (i.m.) route and the other half was injected subcutaneosly (s.c.). Positive control animals were immunized using $5 \mathrm{ml}$ of the conventional BHV-1 vaccine, formulated with inactivated virus and further revaccinated 3 weeks later with the same immunogen. Serum samples were extracted from peripheral blood at different time-points after the initial inoculation.

\subsection{Analysis of antibody response to plant-expressed gDc protein}

Serum samples from mice and cattle inoculated with the plant-derived $\mathrm{gDc}$ were tested by Western blot and ELISA for reactivity against different BHV-1 antigens.

\subsubsection{Western blot}

Recombinant $g D-E c$ proteins were applied to a $12.5 \%$ SDS-PAGE, blotted onto an Immobilon P (Millipore) membrane and then incubated overnight in blotto buffer at $4{ }^{\circ} \mathrm{C}$. The next day, membranes were washed and incubated for $2 \mathrm{~h}$ at $37{ }^{\circ} \mathrm{C}$ with serum samples from immunized cattle diluted $1 / 20$ in the same buffer. Blots were washed again and then incubated with an AP-labeled anti-bovine Ig guinea pig antiserum (Dakkopats) for $1 \mathrm{~h}$ at $37^{\circ} \mathrm{C}$. After extensive washing the reaction was developed by the addition of the NBT/BCIP substrate.

\subsubsection{ELISA for gD-specific antibodies}

Purified recombinant $g D$ - $E c$ diluted in $0.1 \mathrm{M}$ carbonatebicarbonate buffer $\mathrm{pH} 9.6(0.875 \mu \mathrm{g}$ per well) was adsorbed to Immulon II plates (Dynatech) by overnight incubation at $4{ }^{\circ} \mathrm{C}$. Plates then were blocked with PBS-Tween $201 \%$ gelatin (blocking buffer) and subsequently incubated for $1 \mathrm{~h}$ at $37^{\circ} \mathrm{C}$ with mice serum samples and then a secondary anti-mouse-Ig-specific HRP-conjugated antibody (KPL). Primary and secondary antibodies were diluted with the same blocking buffer. The reaction was developed by addition of $o$-phenylenediamine (1,2-benzenediamine) dihydrochloride- $\mathrm{H}_{2} \mathrm{O}_{2}$ in phosphate-citrate buffer $\mathrm{pH} 4.5$ and read $3 \mathrm{~min}$ later at $490 \mathrm{~nm}$ in an MR 500 Microplate Reader (Dynatech). Titers were expressed as $\log 10$ of the reciprocal of the highest serum dilution which gave optical density (OD) readings of at least twice a pool of sera from three animals immunized with extracts of plants inoculated with the empty vector (TMV-30B) at the same dilution.

\subsubsection{ELISA for BHV-1-specific antibodies}

Immulon I plates (Dynatech) were incubated overnight at $4{ }^{\circ} \mathrm{C}$ with partially purified BHV-1 diluted in $0.1 \mathrm{M}$ carbonate-bicarbonate buffer $\mathrm{pH}$ 9.6, as described before [18]. The next day, plates were blocked for $1 \mathrm{~h}$ with PBST $1 \%$ egg albumin, and mice or cattle serum samples were then incubated for $30 \mathrm{~min}$ at $37^{\circ} \mathrm{C}$. After washing, anti-mice-Ig or bovine-Ig-specific HRP-conjugated antibodies were diluted in the same blocking buffer, added to the plates and incubated for $30 \mathrm{~min}$ at $37^{\circ} \mathrm{C}$. The reaction was developed at room temperature by addition of 2,2'-azino-bis(3-ethylbenz-thiazoline-6-sulfonic acid) $-\mathrm{H}_{2} \mathrm{O}_{2}$ in citrate buffer $\mathrm{pH} 5$ and the OD at $410 \mathrm{~nm}$ was measured $20 \mathrm{~min}$ later in an MR 500 Microplate Reader (Dynatech). Titers for mice serum samples were calculated as described for the $\mathrm{gD}$-specific ELISA. Titers for bovine sera were expressed as $\log 10$ of the reciprocal of the highest 
serum dilution which gives OD readings $40 \%$ above a pool of sera (diluted 1:2560) from BHV-1 vaccinated animals.

\subsection{BHV-1 production and purification}

BHV-1 strain Los Angeles (LA) was propagated in Madin Darby bovine kidney (MDBK) cells grown in Eagle's Minimal Essential Medium supplemented with $10 \%$ foetal bovine serum (FBS; Gibco). Confluent monolayers were inoculated with BHV-1 at a multiplicity of infection of 0.1 and the virus was allowed to adsorb for $45 \mathrm{~min}$ at $37^{\circ} \mathrm{C}$ before the addition of fresh culture medium. After a cytopathogenic effect (CPE) was observed, the supernatant was collected and cellular debris was removed by centrifugation at $3000 \times g$ for $30 \mathrm{~min}$. For vaccine formulation, virus was diluted to $10^{7.5}$ tissue-culture infective dose $50 \%$ and then inactivated with BEI [18]. For ELISA, supernatants were pelleted by ultracentrifugation at $120,000 \times g$ for $1 \mathrm{~h}$ at $4 \mathrm{C}$. For in vitro lymphoproliferation assays, virus suspensions were diluted to $10^{5} \mathrm{TCID}_{50} / \mathrm{ml}$, stored at $-70^{\circ} \mathrm{C}$ and immediately before use, inactivated by UV light exposure for $1 \mathrm{~min}$ at $11 \mathrm{~cm}$ from the source (General Electric G875 UV bulbs).

\subsection{In vitro lymphoproliferation assay}

Blood samples were collected by venipuncture with syringes containing heparin. Lymphocyte-enriched cells were isolated from the buffy coats by centrifugation on Lymphoprep $^{\mathrm{TM}}$ (density $1.077 \mathrm{~g} / \mathrm{ml}$; Nycomed Pharma A.S). Cells were then washed twice in RPMI 1640 medium supplemented with $5 \%$ of foetal bovine serum and finally resuspended at a concentration of $2.5 \times 10^{6}$ cells $/ \mathrm{ml}$ in the same culture medium with $10 \%$ FBS. Inactivated BHV-1 virus antigen was diluted 1:100 in RPMI 1640 medium and added to the cultures containing $2.5 \times 10^{5}$ lymphocytes per well, reaching a final volume of $200 \mu \mathrm{l}$ per well. Lymphocytes were incubated for 4 days at $37^{\circ} \mathrm{C}$ in a humidified atmosphere with $5 \% \mathrm{CO}_{2}$. Cell proliferation was measured by addition of $0.4 \mu \mathrm{Ci}\left[{ }^{3} \mathrm{H}\right]$ thymidine per well $20 \mathrm{~h}$ before harvesting. Each test was performed in triplicate. Thymidine uptake for each culture was determined in a liquid scintillation counter (LKB, Wallac, 1219 Rackbeta), and arithmetic means and standard deviations were calculated for triplicate values. Results were obtained as mean counts per minute (cpm) and expressed as stimulation index (SI = mean cpm of cultures in the presence of BHV-1 antigen/mean cpm of cultures without BHV-1 antigen). The threshold value was established at $\mathrm{SI}=3$.

\subsection{Challenge experiments}

Animals were challenged at $210 \mathrm{dpi}$ using BHV-1 virus (LA strain) suspended in Eagle's medium at a concentration of $10^{7.5}$ TCID $_{50}$. A volume of $1.5 \mathrm{ml}$ of the inoculum was administered into each nostril of individual animals by aerosol exposition using an ultrasonic nebulizer
(Electrolab AP-300). Prior to challenge, animals were clinically examined, rectal temperature recorded and blood samples and nasal swabs were collected to establish baseline values. Nasal swabs were obtained by inserting tampons into the ventral meatus of the nasal passage and immediately dipping them in MEM containing $5000 \mathrm{IU}$ penicillin/ml, $2500 \mu \mathrm{g}$ streptomycin/ml and $10 \mu \mathrm{g}$ amphotericin $\mathrm{B} / \mathrm{ml}$. Samples were collected 2, 4, 5, 7, 9, 11, 12, and 15 days post-challenge (dpch). For virus detection, serial dilutions of the nasal fluids were immediately inoculated onto MDBK cell monolayers cultured in 96 well plates. Monolayers were inspected for $48 \mathrm{~h}$ for the appearance of cytopathological effect and results were expressed as $\mathrm{TCID}_{50} / \mathrm{ml}$ of nasal fluid. Cattle were clinically examined for 15 consecutive days after challenge and evaluated for the presence of anorexia, increase of body temperature, rhinitis and vulvovaginitis.

\section{Results}

\subsection{Expression of recombinant $g D c$ in plants}

Infective RNA transcripts were synthesized from the TMV-gDc and TMV-30B plasmids and utilized for inoculation of $N$. benthamiana. Infected plants were isolated in growth chambers and inoculated leaves were harvested 1 week after infection. In order to analyze the production of the recombinant $\mathrm{gDc}$, total proteins were extracted, separated in a $12.5 \%$ SDS-PAGE and transferred to a nitrocellulose membrane. An equivalent amount of protein extracted from $6 \mathrm{mg}$ of fresh leaf tissue was loaded for all of the TMV-gDc, TMV-30B and mock infected samples. A sample with $50 \mathrm{ng}$ of $g D-E c$ protein served as a positive control. Blots were analyzed by a Western assay using a mouse anti- $g D-E c$ as the probe. Results in Fig. 2 show the presence of a positive signal with similar electrophoretic

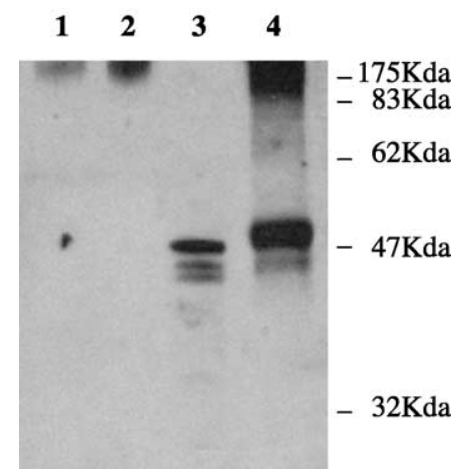

Fig. 2. Expression of the recombinant gDc protein in plants. Western blot of plant extracts and recombinant gDc developed using a mouse polyclonal serum against the $g D-E c$. Foliar extracts from mock infected (lane 1), TMV-30B inoculated (lane 2), and TMV-gDc inoculated (lane 4) plants were blotted together with $0.05 \mu \mathrm{g}$ of $g D$-Ec (lane 3). All plant samples derived from $6 \mathrm{mg}$ of fresh leaves. Position of protein molecular weight standards is indicated on the right side of the blot. 
mobility in both TMV-gDc and $g D-E c$ lanes. The amount of the recombinant $\mathrm{gDc}$ was estimated at about $15-20 \mu \mathrm{g} / \mathrm{g}$ of fresh tissue. No signal was detected for the protein extracts from TMV-30B or mock infected leaves.

\subsection{Induction of an anti-BHV-1 response in mice immunized with crude foliar extracts inoculated with the $T M V-g D c$}

Mice were immunized using an oil-based vaccine containing $0.2 \mathrm{~g}$ of fresh leaf tissue per dose. Serum samples were recovered at different times post-immunization with the purpose of measuring reactivity against BHV-1 antigens. The recombinant $g D-E c$ was utilized as the antigen in an indirect ELISA to monitor the titre of anti-gDc antibodies induced in the mice after injection of the plant extracts. The results in Fig. 3a and $\mathrm{b}$ demonstrate that all animals immunized with a single dose of the plant-produced gDc developed a specific and significant humoral response to both gDc and BHV-1, presenting a kinetic that clearly resembles that of the animal immunized with a conventional vaccine for up to $80 \mathrm{dpi}$.
3.3. Anti-BHV-1 humoral immune responses in cattle immunized with crude foliar extracts inoculated with the $T M V-g D c$

Cows serologically negative for BHV-1 were immunized with single vaccine doses consisting of a total of $5 \mathrm{~g}$ of fresh leaf tissue infected with either TMV-gDc or TMV-30B. Western blot analysis revealed, after two vaccinations, that the plant-derived gDc was able to induce a specific antibody response in cattle directed against recombinant $g D-E c$. Moreover, the antibody reactivity of the plant-gDc immunized animals was similar to that of a pool of BHV-1 vaccinated cattle. As expected, cows inoculated with the control TMV-30B did not have antibodies that reacted against the BHV-1-specific bands (Fig. 4). An ELISA using whole virus as antigen was utilized to evaluate the antibody response directed against whole BHV throughout the experimental period. These results confirmed that cattle immunized with the plant-produced gDc were also able to develop a specific and significant response against the native antigen (Fig. 5). To control for possible contact of the test animals with BHV-1
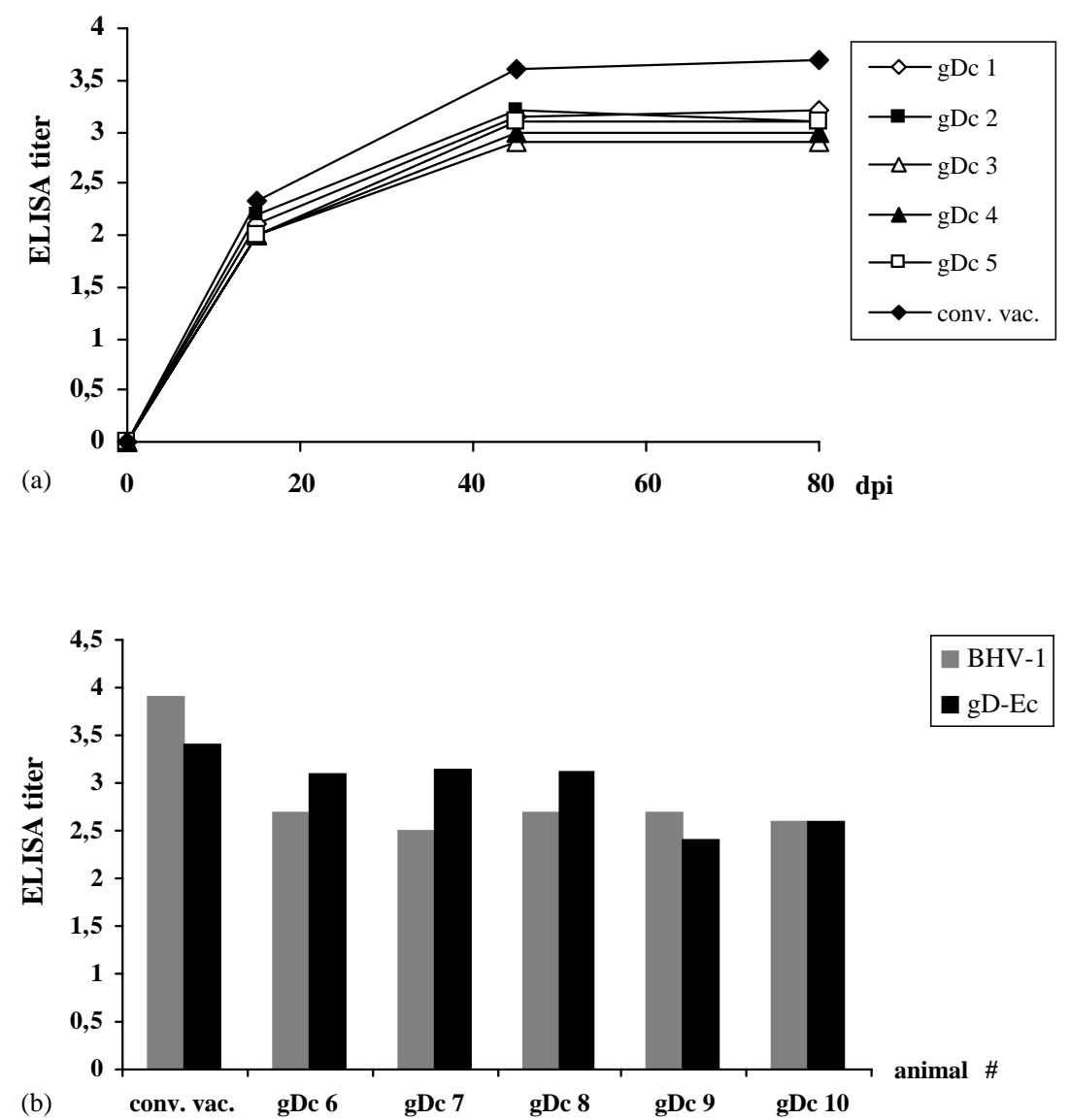

Fig. 3. Humoral immune response induced by the plant-produced gDc in mice. Titers are expressed as described in Section 2. (a) Samples were tested by ELISA for presence of anti BHV-1 gD antibodies. Animals were immunized with a conventional BHV-1 vaccine (conv. vac.) or immunogens containing fresh leaf extracts from plants inoculated with TMV-gDc (gDc1-gDc5). The titers are expressed relative to those of the control animals injected with TMV-30B, (b) Comparative response to BHV-1 whole particles and gD protein at 70 dpi. Mice were immunized as indicated above and serum samples were tested by ELISA using either partially purified BHV-1 particles (grey bars) or gD-Ec (black bars) as antigens adsorbed to the plates. 


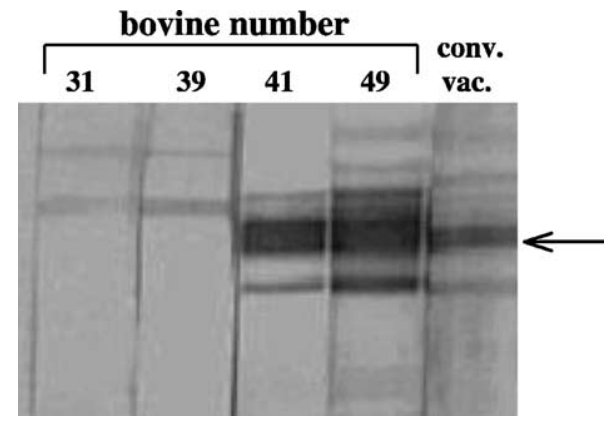

Fig. 4. Immune response ( $40 \mathrm{dpi})$ of cattle inoculated with the recombinant gDc produced in plants. Sera were tested by Western blot against the purified gD-Ec. Cows 31 and 39 were immunized with foliar extracts from TMV-30 infected plants and animals 41 and 49 received extracts from TMV-gDc infected plants. The positive control represents a pool of sera from five cows immunized with a conventional BHV-1 vaccine (conv. vac. \#1-5). The arrow indicates the position of the $g D-E c$ protein.

in the field during the course of the experiment, a commercial ELISA system was used to test the reactivity of all the animals against the gE antigen of BHV-1 (data not shown).

\subsection{Anti-BHV-1 cellular immune responses in cattle immunized with crude extracts from leaves inoculated with the TMV-gDc}

Lymphocyte samples from PBMC were obtained at $80 \mathrm{dpi}$ from cattle injected with crude foliar extracts from TMV-gDc and TMV-30B infected plants. An additional animal inoculated with a commercial vaccine was also included in the experiment as positive control. In vitro lymphoproliferation was measured by assessing the amount of label incorporated by the cells in the presence of inactivated BHV-1 particles after $96 \mathrm{~h}$ of culture. Lymphocytes from animals inoculated with the plant-produced $\mathrm{gDc}$ exhibited positive proliferation indexes (mean SI $=3.6$ and 7.0) comparable to the BHV-1 vaccinated cow (mean SI $=4.9$ ). Cows inoculated with the TMV-30B-infected foliar extracts presented a mean $\mathrm{SI}=1.1$, under the threshold for positive responses $(\mathrm{SI}=3.0)$ (Fig. 6).

\subsection{Challenge experiments in cows vaccinated with $T M V-g D c$ inoculated foliar extracts}

Finally, to test for the possible protective effect of the recombinant vaccines, animals vaccinated with the different foliar extracts were challenged with infective BHV-1 at 210 dpi. Protection levels obtained were assessed from the amount of excreted virus in nasal fluids (Fig. 7). Unvaccinated control animals and cows vaccinated with TMV-30B alone showed similar patterns of virus shedding. Interestingly, virus shedding was delayed up to 5 days in cattle that received plant extracts containing gDc and virus titers were reduced between 100 and 10,000 times in these animals. Moreover, two of the BHV-1 vaccinated animals (conv. vac. \#2 and \#4) showed similar and even higher virus excretion titers than the plant-gDc inoculated cows. These results were affirmed with the observation that clinical symptoms appeared later and were milder in the plant-gDc vaccinated animals (data not shown).

\section{Discussion}

We have demonstrated here that a plant viral based vector can be used to produce sufficient quantities of an animal viral antigen in crude plant extracts to be useful for effectively immunizing animals against animal viral pathogens.

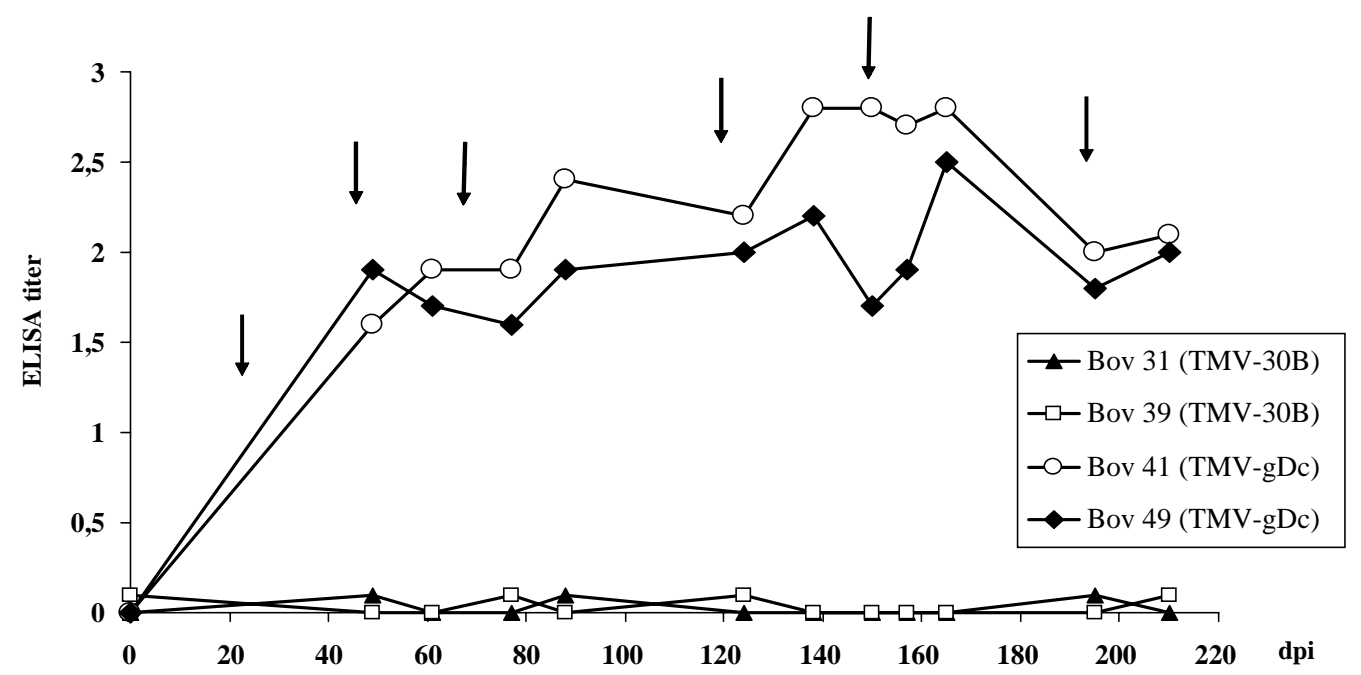

Fig. 5. Humoral immune response induced in cattle injected with plant extracts. (a) Serum samples were extracted at different time-points and tested by ELISA using whole BHV-1 particles as antigen. Primary vaccination and subsequent booster injections (indicated by arrows) were performed using $5 \mathrm{~g}$ of fresh leaf tissue from plants inoculated with TMV-gDc (animals 41 and 49) or TMV-30B (animals 31 and 39). 


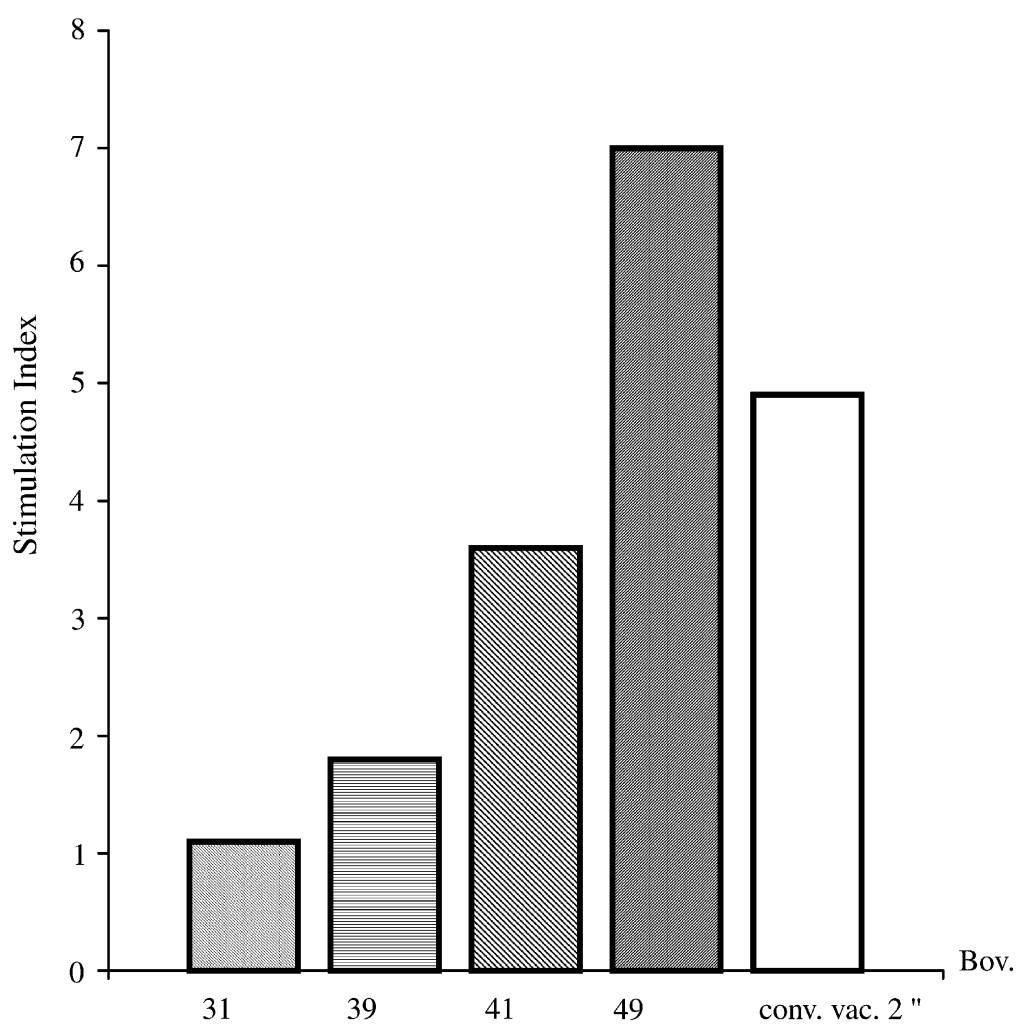

Fig. 6. Cellular immune response induced in cattle to the plant-produced gDc. Lymphocyte-enriched cell cultures were prepared from PBMC at 80 dpi. Blood cell samples were extracted from cattle immunized with crude foliar extracts from plants infected with TMV-gDc (bovines 41 and 49), TMV-30B (animals 31 and 39), or from one animal injected with the BHV-1 conventional vaccine (conv. vac. \#2). For each animal, triplicate cell cultures were incubated with or without the specific BHV-1 antigen. Stimulation indexes in each case were determined as a ratio between the arithmetic means of cultures with and without antigen ( $\mathrm{SI}=3$ are considered positive).

Previously, we demonstrated that protective immunity could be produced in mice injected with plant extracts containing VP1 of foot-and-mouth disease virus expressed from the same TMV vector [15]. Together, these two examples, using two very different proteins derived from unrelated RNA and DNA virus candidates, provide good evidence that animal viral proteins expressed in plants retain sufficient structural integrity to be useful for eliciting immunologically useful responses in test animals.

First, our results showed quite convincingly that the TMV vector carrying a cytoplasmic form of the glycoprotein D subunit of bovine herpesvirus was able to direct the synthesis of a protein of the expected electrophoretic mobility (ca. $55 \mathrm{kDa}$ ) and antigenic identity at concentrations estimated in the range of $20 \mu \mathrm{g} / \mathrm{g}$ of fresh tissue. The protein appeared to be stable in the plant extracts and slightly larger in size than the E. coli-expressed $\mathrm{gD}$. We assessed if the recombinant plant-produced gDc retained a proper conformation by testing its antigenic properties by performing a series of immunological experiments in both an animal model and the natural host.

The antigenicity of the recombinant $\mathrm{gDc}$ was initially studied in a murine model. A group of 10 animals received a single dose of a vaccine formulated with crude extracts containing approximately $2 \mu \mathrm{g}$ of the plant-gDc. All the animals developed a specific and sustained immune response against both the $\mathrm{gDc}$ and the BHV-1, for up to $80 \mathrm{dpi}$. This response was comparable to that registered for mice vaccinated with the conventional immunogen, indicating that the plant-gDc was immunogenic in mice and retained B-cell epitopes that were also present in the native virus particle. Analysis of the BHV-1-specific IgG isotype also demonstrated the presence of predominant levels of both $\mathrm{IgG} 1$ and $\mathrm{IgG} 2 \mathrm{a}$ and to a lesser extent IgG2b and IgG3 (data not shown). In contrast, animals immunized with the inactivated-virus vaccine presented only significant levels of IgG1. These results indicate the presence and functionality of T-cell epitopes in this plant-produced gDc immunogen. This is consistent with the high and persistent level of specific antibodies induced by this protein.

Based on the positive results in mice, we then examined the immune response induced by the plant-gDc in the natural bovine host. Cattle were immunized using the same crude immunogen, but they received a larger dose and a greater number of booster doses because of the large size of the animals. Both of the cows immunized with the gDc containing plant extracts developed significant ELISA titers against the recombinant $g D-E c$ by $40 \mathrm{dpi}$. This anti-gDc antibody response strongly correlated with the specific anti BHV-1 humoral response measured at $50 \mathrm{dpi}$ and the positive 

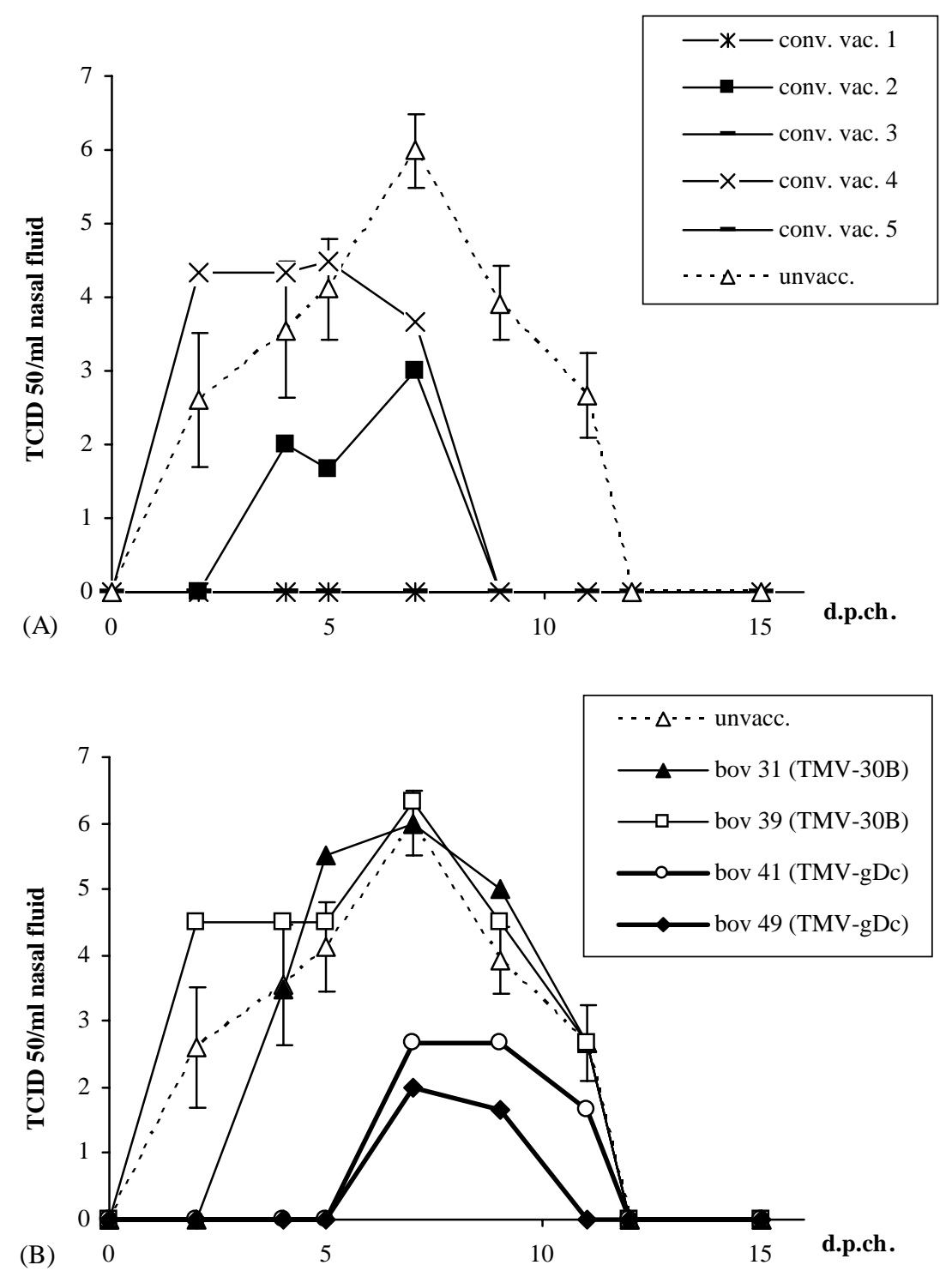

Fig. 7. Virus excretion in cows after challenge with infective BHV-1. Cattle were challenged at 210 dpi and the presence of infective virus in nasal excretions was assessed for each animal. The titers obtained are presented separately for each animal, except for unvaccinated controls. Both panels: the unvaccinated control results represent the mean titer ( \pm S.D.) obtained from four animals. Panel A: animals vaccinated with the BHV-1 conventional vaccines (animals 1-5) or with extracts from plants. Panel B: animals vaccinated with extracts from plants inoculated with TMV-30B (bovines 31 and 39) or TMV-gDc (bovines 41 and 49).

cellular immune response evaluated at $80 \mathrm{dpi}$. Moreover, cattle immunized with the plant-produced $\mathrm{gDc}$ presented good levels of protection to viral challenge. These results were consistent with the observed cellular response, as it is generally established that stimulation of the cellular immunity plays a central role in the protection against the BHV-1 infection [27]. Both the emergence of symptoms and detection of virus in nasal excretions were significantly delayed and shortened in these animals compared with controls. Importantly, gDc-vaccinated cattle presented much less severe symptoms and virus shedding in nasal fluids was reduced by $2-4 \operatorname{logs}$ in comparison with negative controls. Conventionally vaccinated animals showed variable levels of pro- tection, as previously reported [26]. While three of them did not show significant virus shedding, the other two showed an early detectable virus excretion. A most interesting result was that protection levels reached in cows immunized with the plant-derived antigens - in terms of length, delay and reduction of virus excretion-were similar to or even better than some of animals that received the conventional vaccine. Our results contrast with previous reports which were unsuccessful at eliciting a level of protective response as presented here when they used an unglycosylated form of the BHV $\mathrm{gD}$ produced in E. coli [22].

This is the first report showing induction of protective antibodies in cattle immunized with a plant-produced BHV-1 
antigen. In conclusion, we have now demonstrated that the TMV-30B vector is suitable for production of proteins with moderately high molecular weight such as gDc. The immunological characterization of the protein showed that a significant part of the native gD conformation was retained in the recombinant plant protein. These results, along with those reported previously for FMDV demonstrate that a structural protein of an RNA virus and an external membrane protein of a DNA virus can be expressed in plants in sufficient quantity and of sufficient quality to be useful for eliciting protective antibodies in vaccinated animals. We recognize that that this relatively simple technology needs to be further refined in order to be put into practice for production of low cost and biologically safe animal vaccines. Although optimization of the technology for commercial production of useful vaccines utilizing this technology is beyond the scope of this study, a recent report suggests that this goal could be effectively accomplished through a commercial partnership (personal communication, Greg Pogue, Large Scale Biology Corporation). In this era of concern over the deliberate release of animal pathogens for the purposes of bioterrorism, it would seem prudent for regulatory agencies to further explore this relatively inexpensive approach for producing agriculturally important vaccines.

\section{Acknowledgements}

We would like to thank Jill Hyslop and Marta Radolci for excellent technical assistance.

\section{References}

[1] Giddings G, Allison G, Brooks D, Carter A. Transgenic plants as factories for biopharmaceuticals. Nat Biotechnol 2000;18:1151-5.

[2] Turpen TH, Reinl SJ, Charoenvit Y, Hoffman SL, Fallarme V, Grill LK. Production of recombinant proteins in plants: pharmaceutical applications, The Soc. Exp. Biol., Canterbury, UK. J Exp Bot 1997;48(Suppl):12-21.

[3] Mason HS, Warzecha H, Mor T, Arntzen CJ. Edible plant vaccines: applications for prophylactic and therapeutic molecular medice. Trends Mol Med 2002;8:324-9.

[4] Arakawa T, Chong DKX, Langridge WHR. Efficacy of a food plant based oral cholera toxin B subunit vaccine. Nat Biotech 1998;16: 292-7.

[5] Mason HS, Lam DM-K, Arntzen CJ. Expression of hepatitis B surface antigen in transgenic plants. Proc Natl Acad Sci USA 1992;89:11745-9.

[6] Mason HS, Ball JM, Shi JJ, Jiang X, Estes MK, Arntzen CJ. Expression of Norwalk virus capsid protein in transgenic tobacco and potato and its oral immunogenicity in mice. Proc Natl Acad Sci USA 1996;93:5335-40.

[7] Gómez N, Carrillo C, Parra F, Salinas J, Borca MV, Escribano JM. Expression of immunogenic glycoprotein $\mathrm{S}$ polypeptides from transmissible gastroenteritis coronavirus in transgenic plants. Virology 1998;249:352-8.

[8] Lacomme C, Smolenska L, Wilson TM. Genetic engineering and the expression of foreign peptides or proteins with plant virus-based vectors. In: Setlow JK, editor. Genetic Engineering. New York: Plenum Press; 1998. p. 225-37.
[9] Johnson J, Lin T, Lomonosoff G. Presentation of heterologous peptides on plant viruses: genetics, structure and function. Annu Rev Phytopathol 1997;35:67-86.

[10] Scholthof HB, Scholthof K-BG, Jackson AO. Plant virus gene vectors for transient expression of foreign proteins in plants. Annu Rev Phytopathol 1996;34:299-323.

[11] Yusibov V, Shivprasad S, Turpen TH, Dawson W, Koprowski H. Plant viral vectors based on tobacco mosaic viruses. Curr Top Microbiol Immunol 1999;240:81-94.

[12] Kumagai MH, Turpen TH, Weinzettl N, della-Cioppa G, Turpen AM, Donson J, et al. Rapid, high-level expression of biologically active alpha-trichosanthin in transfected plants by an RNA viral vector. Proc Natl Acad Sci USA 1993;90:427-30.

[13] McCormick AA, Kumagai MH, Hanley K, Turpen TH, Hakim I, Grill LK, et al. Rapid production of specific vaccines for lymphoma by expression of the tumor-derived single-chain Fv epitopes in tobacco plants. Proc Natl Acad Sci USA 1999;96:703-8.

[14] Turpen TH, Reinl SJ, Charoenvit Y, Hoffman SL, Fallarme V, Grill LK. Malarial epitopes expressed on the surface of recombinant tobacco mosaic virus. Biotechnology (NY) 1995;13:53-7.

[15] Wigdorovitz A, Perez Filgueira DM, Robertson N, Carrillo C, Sadir AM, Morris TJ, et al. Protection of mice against challenge with foot and mouth disease virus (FMDV) by immunization with foliar extracts from plants infected with recombinant tobacco mosaic virus expressing the FMDV structural protein VP1. Virology 1999;264: $85-91$.

[16] Shivprasad S, Pogue GP, Lewandowski DJ, Hidalgo J, Donson $\mathrm{J}$, Grill LK, et al. Heterologous sequences greatly affect foreign gene expression in tobacco mosaic virus-based vectors. Virology 1999;255:312-23.

[17] Kahrs RF. Infectious bovine rhinotracheitis: a review and update. J Am Vet Med Assoc 1977;171:1055-64.

[18] Engels M, Ackerman M. Pathogenesis of ruminant herpesvirus infections. Vet Microbiol 1996;53:3-15.

[19] Frerichs GN, Woods SB, Lucas MH, Sands JJ. Safety and efficacy of live and inactivated infectious bovine rhinotracheitis vaccines. Vet Rec 1982;111:116-22.

[20] Yates WD. A review of infectious bovine rhinotracheitis, shipping fever pneumonia and viral-bacterial synergism in respiratory disease of cattle. Can J Comp Med 1982;46:225-63.

[21] Babiuk LA, L'Italien J, van Drunen Littel-van den Hurk S, Zamb T, Lawman JP, Hughes G, et al. Protection of cattle from bovine herpesvirus type I (BHV-1) infection by immunization with individual viral glycoproteins. Virology 1987;159:57-66.

[22] van Drunen Littel-van den Hurk S, Parker MD, Massie B, van den Hurk JV, Harland R, Babiuk LA, et al. Protection of cattle from BHV-1 infection by immunization with recombinant glycoprotein gIV. Vaccine 1993;11:25-35.

[23] Zhu X, Wu S, Letchworth III GJ. Yeast-secreted bovine herpesvirus type 1 glycoprotein $D$ has authentic conformational structure and immunogenicity. Vaccine 1997;15:679-88.

[24] Bosch JC, De Jong MC, Franken P, Frankena K, Hage JJ, Kaashoek $\mathrm{MJ}$, et al. An inactivated gE-negative marker vaccine and an experimental $\mathrm{gD}$-subunit vaccine reduce the incidence of bovine herpesvirus 1 infections in the field. Vaccine 1998;16:265-71.

[25] Zamorano P, Taboga O, Dominguez M, Romera A, Puntel M, Tami C, et al. BHV-1 DNA vaccination: effect of the adjuvant RN-205 on the modulation of the immune response in mice. Vaccine 2002;20: 2656-64.

[26] Romera SA, Hilgers LA, Puntel M, Zamorano PI, Alcon VL, Dus Santos MJ, et al. Adjuvant effects of sulfolipo-cyclodextrin in a squalane-in-water and water-in-mineral oil emulsions for BHV-1 vaccines in cattle. Vaccine 2000;19:132-41.

[27] van Drunen Littel-van den Hurk S, Tikoo SK, Liang X, Babiuk LA. Bovine herpesvirus-1 vaccines. Immunol Cell Biol 1993;71:405-20. 